distribution of the comparison stars can be obtained. The spectrum tracings have been handled so that the measurements made refer to the continuum as seen on the tracing. Since the resolution is only 7 Angstroms, it is impossible to correct for faint absorption lines; this correction could be made with the help of high dispersion spectra.
An attempt is being made to compare the theoretical and observed energy distributions at various phases by methods which have in recent years been applied to the six-color photometric results.

\title{
COMMENTS ON THE PRESENT STATUS OF THE PULSATION THEORY
}

\section{By CHARLES A. WHITNEY}

Instability against radial pulsation is discussed briefly from a thermodynamic point of view. Two possible explicit causes are outlined: variations of nuclear generation in the stellar interior; the production of a "heat engine" in the external layers through the action of variable opacity and heat storage.

An encouraging semi-quantitative test of the second alternative is discussed.
A model for the atmospheric motions of $\mathrm{W}$ Virginis is presented and observational tests are suggested. Theoretical problems are indicated whose solutions are required for further progress in the interpretation of the spectra of classical Cepheids and W Virginis stars.

Smithsonian Astrophysical Observatory, Cambridge, Mass.

\section{ABSTRACTS \\ OF PAPERS PRESENTED AT THE NINETY-NINTH MEETING OF THE AMERICAN ASTRONOMICAL SOCIETY, INDIANAPOLIS, IND., DECEMBER 27-30, 1957}

\section{Aller, Lawrence H. Magnetic stellar envelopes and planetary nebulae.}

It has been pointed out by Minkowski and others that the structural appearance of many planetary nebulae strongly suggest the presence of magnetic fields. It seems unlikely that such magnetic fields are produced $a b$ initio in the nebular shell. Rather, they must have existed in the outer envelope of the parent star. Certain red giant stars with magnetic fields may evolve in such a way that the expansion of the shell is largely governed by the presence of such a field. Magnetic effects may actually be more important than gas pressure differentials and radiation pressure in controlling the evolution of a planetary nebula. The suggestion that combination variables such as AX Persei, BF Cygni, and $Z$ Andromedae may be the immediate parents of the planetary nebulae now appears particularly tempting. Not all such stars necessarily are presumed to evolve into planetary nebulae.

University of Michigan Observatory,
Baum, W. A.; Ford, W. Kent Jr. and Hall, John S. Recent astronomical tests of thin-film image converters.

During the past three years the astronomical practicability of a special type of electronic image converter tube has been explored. This tube operates essentially by A. Lallemand's method (i.e., electrons ejected from a photocathode are accelerated and focused directly onto a nuclear-track emulsion) but a very thin barrier film or membrane has been added to prevent gas molecules exuded by the emulsion from reaching the photocathode and poisoning it. Electrons of several kilovolts energy can pass through this film but gas molecules cannot. Attention has been confined to designs which are potentially capable of being produced commercially, because the ultimate value of any technique in this field will depend upon its general availability to observatories everywhere.

In August and November, I957, a number of electron-produced images on Ilford $\mathrm{G}_{5}$ plates were obtained at the 40-inch telescope of the 
Naval Observatory at Flagstaff, Arizona. The useful field of the Farnsworth tubes under test has a diameter of about $\mathrm{I} 8 \mathrm{~mm}$, which corresponds to 9 minutes of arc at the focus of the telescope.

The components of double stars were separated on the plate when the distance between their centers was 170 microns; this corresponds to an angular separation of 5 seconds of arc. It is important that the gap between the barrier film and the $\mathrm{G}_{5}$ emulsion should be very small so that the scattering of the photoelectrons by the barrier film will not result in a serious loss of image resolution. A gap of 40 microns has been successfully used at the telescope but attempts in the laboratory to improve the resolution by bringing the film into actual contact with the $\mathrm{G}_{5}$ emulsion by means of electrostatic deflection have given promising results.

A number of 30-second exposures were made on M36 with a tube of low sensitivity and compared with direct photographs of $\mathrm{M}_{3} 6$ secured on 103a-D emulsions. Microphotometer tracings indicated that the signal-to-noise performance with the image tube was roughly on a par with that obtained by short-exposure photography. The same image density would have been obtained in a one-second exposure if the cathode of the image tube had been of average sensitivity.

In order to avoid excessive background emission, the cathode of the image tube was cooled and accelerating potentials of $10 \mathrm{kv}$ or less were used. Minor changes in tube design and more efficient cooling should permit the observer to extend the useful exposure time well beyond 30 seconds.

The Formvar-aluminum barrier films used in this application had a total thickness of $700 \mathrm{~A}$. Ford has made a study of the physical properties of self-supported films and has succeeded in making films which will withstand the high temperatures required in image-tube processing and which are strong, flat, uniform, and free of pinholes. The two tubes used in these experiments were operated for three days without substantial loss in sensitivity.

$$
\begin{array}{r}
\text { Mount Wilson and Palomar Observatories, } \\
\text { Pasadena, Calif. } \\
\text { and Department of Terrestrial Magnetism, } \\
\text { Carnegie Institution of Washington } \\
\text { and U. S. Naval Observatory, } \\
\text { Washington, D. C. }
\end{array}
$$

Buscombe, William and Morris, Pamela M. Southern stars of high velocity.

In connection with the choice of observing programs for the determination of radial veloci- ties with the Zeiss spectrograph at the Newtonian focus of the 74-inch reflector, a finding list of southern high-velocity stars is being prepared for press.

Tangential velocities were first computed for all stars south of the celestial equator, for which reliable trigonometric parallaxes (i.e., $>0$ o.or) are available. The stars whose space velocities (corrected for a solar motion component of $20 \mathrm{~km} / \mathrm{sec}$ toward R.A. $270^{\circ}$, Dec. $+30^{\circ}$ relative to the nearby stars) exceed $60 \mathrm{~km} / \mathrm{sec}$ have been included, plus the stars of which the corrected tangential or radial velocities separately exceed $50 \mathrm{~km} / \mathrm{sec}$.

Pending the procurement of more complete data, many stars are also listed for which the uncorrected proper motion exceeds 0 ". 5 annually. They are chiefly included for the purpose of drawing attention to the gaps which must be filled in the observational data, before a complete statistical analysis of the kinematics of stars in the high-velocity groups can be made, extending to any limits fainter than visual magnitude 5.5 .

No attempt is being made at the moment to introduce photoelectric measures of stellar magnitude, or revised spectral classifications, as those fragmentary data would detract from the homogeneity of the Harvard system of photographic photometry and Henry Draper catalogue types. The use of spectroscopic parallaxes has been expressly rejected, as these have proved unreliable in previous surveys, particularly for stars of abnormal population groups. Objects of types such as RR Lyrae variables, globular clusters and planetary nebulae, which obviously belong to Population II, are omitted.

Observers are invited to make available any relevant unpublished data on parallaxes, proper motions or radial velocities which may enhance the usefulness of the finding list, and spare future observers the unfruitful effort of duplicating their work.

Mount Stromlo Observatory Canberra, Australia

\section{Cameron, A. G. W. Mechanism of neutron production in supernova explosions.}

If the main energy source giving rise to the exponential tails in the light curves of Type I supernovae is the spontaneous fission of $C f^{254}$, then nuclei of medium atomic weight in these stars must have captured an average of 200 or more neutrons. It has been suggested that in the 